www.jmscr.igmpublication.org

Impact Factor 5.84

Index Copernicus Value: 83.27

ISSN (e)-2347-176x ISSN (p) 2455-0450

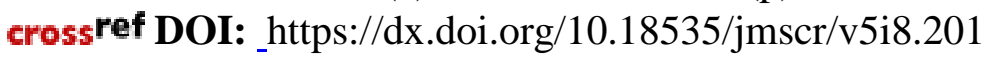

Journal Of Medical Science And Clinical Research

\title{
Echocardiographic evaluation of cardiac function in hypothyroidism
}

\author{
Authors \\ Dr Sunita D. Kumbhalkar*1, Dr Shakti P. Choudhury ${ }^{2}$ \\ ${ }^{1}$ Associate Professor, ${ }^{2}$ Junior Resident \\ Department of Medicine, Government Medical College, Nagpur, Maharashtra, India- 440001 \\ Corresponding Author \\ Dr Sunita Kumbhalkar* \\ Email: skumbhalkar@gmail.com
}

\begin{abstract}
Aim and Objectives: To study the clinical profile and various echocardiographic changes in hypothyroid patients and compare these changes between hypothyroid patients and controls.

Methods: We included 32 consecutive first time detected hypothyroid patients diagnosed through thyroid profile and 15 healthy age and sex matched controls. The clinical electrocardiographic and echocardiographic features of the study group were compared to their respective age and sex matched healthy controls.
\end{abstract}

Results: In current study, most of the patients (56.25\%) had presented with pre-hypertension stage. Hypothyroid patients had significantly raise TSH and low T3 and T4 and also had raised serum cholesterol, triglyceride and LDL concentration as compared to controls. The sinus bradycardia was commonest electrocardiogram abnormality being present in $43.75 \%$ patients followed by low voltage complexes (18.75\%), ST-T changes (15.62\%) and right bundle branch block (3.1\%) while $18.75 \%$ had normal electrocardiogram. There was no statistical difference in basic two dimensional echocardiographic parameters between two groups. We found significant reduction in early diastolic filling (E) with augmented late diastolic filling (A) resulting in decrease E/A ratio suggesting significant diastolic dysfunction associated with significant reduction in, peak early diastolic velocity (E'), raised peak late velocity ( $A$ ') and increased E/E' in both overt and subclinical hypothyroid patients. IVRT and DT were also prolonged. Increased interventricular septal and posterior wall thickness was also found in overt hypothyroid along with pericardial effusion when compared to subclinical and controls. There was no significant difference observed in ejection fraction and systolic velocity between two groups.

Conclusion: This study demonstrated that diastolic function was impaired in patients with hypothyroidism. Doppler-echocardiography may be considered a reliable method for a cross-sectional and longitudinal assessment of left ventricular diastolic function in patients with hypothyroidism.

Keywords: Electrocardiography; Doppler-Echocardiography; Hypothyroidism; Diastolic filling; Systolic velocity, Interventricular septal.

\section{Introduction}

Hypothyroidism is the most common pathological hormone deficiency and it associated with clinically significant cardiovascular changes. Overt hypothyroidism refers to cases in which serum thyrotropin (TSH) concentration is elevated 
and serum T4 is below the reference range while subclinical hypothyroidism defined as an elevated serum TSH value, associated with a serum free T4 within the reference range. However hypothyroiddism produces a decrease in myocardial contractility, pericardial effusion and increase in left ventricular mass and prolonged duration of contraction and relaxation. The ejection fraction and cardiac reserve are only slightly diminished ${ }^{[1]}$. The diastolic parameters depend upon cytosolic calcium concentration modulated by sarcoplasmic reticulum, ATP dependent calcium ${ }^{[2]}$. Calcium transport is controlled by thyroid hormones ${ }^{[3]}$. Hence, diastolic dysfunction can occur in patients with subclinical hypothyroidism. This diastolic impairment may be a prelude to systolic dysfunction. The cardiac complications of long standing hypothyroidism are serious if are not diagnosed properly earlier. Also high index of suspicion has to be there for diagnosis of subclinical hypothyroidism, as symptoms are vague. As a non-invasive method, echocardiography can play important role in recognizing the cardiac pathology as well as to follow up effect of the therapy ${ }^{[4]}$.

Most of cardiac manifestations are reversible with adequate and timely thyroid therapy. The aim of this study was to assess echocardiography changes in patients with untreated first time detected hypothyroid states.

\section{Materials and Methods}

The present study included total 32 newly diagnosed and untreated hypothyroid patients and 15 healthy age and sex matched controls. The 32 patients had presented to us with nonspecific complaints such as fatigue, mild weight gain and depressive feelings or with overt symptoms and signs of thyroid hormone deficiency, therefore thyroid hormone assay was done in all these patients. The hypothyroidism was diagnosed in those cases, who had serum TSH values above normal or with low T3 and T4 level. Free serum T3 and T4 levels could not be done due to financial constraints. Informed consent from study group (hypothyroid patient) and controls was taken prior to inclusion. Patient with clinically evident cardiovascular disease, diabetes mellitus, renal failure, pregnant women, patients with chronic pulmonary disease, severe anaemia or any other endocrinal disorder, patients taking medicines that could alter cardiac functions like amiodarone, beta blockers, calcium channel blockers etc, conditions rendering myocardium stiff like amyloidosis, haemachromatosis, sarcoidosis, alcoholism etc, condition such as presence of any chronic medical and surgical condition which may independently affect the left ventricular function adversely, conditions altering level of total thyroid hormone level such as nephritic syndrome, patients on oral contraceptive pills, drugs (phenytoin, NSAIDS, carbamazepine), pregnancy were excluded from the study.

All patients were evaluated for their symptomatology and underwent detail physical and clinical examination. After clinical and laboratory diagnosis hypothyroid patients were grouped into 1) overt hypothyroid, 2) subclinical hypothyroid. Patients in both the groups were then subjected for electrocardiographic and echocardiographic assessment.

Echocardiography was performed with machine Philips HD $11 \times$ E with a S 4-2 probe. Baseline Mmode quantitative analysis was performed as per the guidelines laid down by the American Society of Echocardiography [130]. Parameters included were 1) dimensions of Aorta (AO) and left atrium (LA) in $\mathrm{mm}, 2$ ) left ventricular end systolic [LVID(s)] and end diastolic [LVID (d)] dimensions in $\mathrm{mm}, 3$ ) interventricular septum (IVS) and posterior wall (PW) in $\mathrm{mm}$. Patients were evaluated by two dimension echocardiography for presence of Regional Wall motion abnormality (RWMA), any regurgitant lesion, pericardial effusion and ejection fraction $(\mathrm{EF} \%)$. Then the Doppler echocardiography was performed. The LV inflow pattern was obtained by keeping the sample volume at the tip of mitral leaflets. 
Echocardiographic parameters were calculated in both the hypothyroid patients and healthy age and sex matched controls. To decrease intra-observer variability echocardiographic values were presented as the average of three measurements compared with the controls.

\section{Statistical Analysis}

Continuous variables (laboratory parameters, MMode parameters and Doppler parameters) were presented as Mean \pm SD. Categorical variables were expressed in actual numbers and percentage. Continuous variables were compared by performing unpaired t-test. Categorical variables were compared by chi2-square test. For small number, fisher exact test was applied wherever applicable. $\mathrm{p}<0.05$ was considered as statistical significance. Statistical software STATA version 10.0 was used for statistical significance.

\section{Observations and Results}

Thirty two hypothyroid patients were studied and compared with fifteen healthy age and sex matched controls. The mean age of study group was 40.9 \pm 8.73 years, ranging from 23-60 years with female predominant $(93.75 \%)$. Within the study group $18 \quad(56.2 \%)$ were subclinical hypothyroid and all were females while 14 $(43.75 \%)$ were overt hypothyroid of which only two were male patients. Table 1 show the general symptoms of hypothyroid patients.

Table 1: Distribution of hypothyroid patients according to general symptoms

\begin{tabular}{|l|c|c|c|}
\hline Symptoms & Male & Female & Total \\
\hline Weight gain & 2 & 18 & $20(62.5 \%)$ \\
\hline Fatigability & 1 & 18 & $19(59.37 \%)$ \\
\hline Dry skin & Nil & 13 & $13(40.62 \%)$ \\
\hline Constipation & 1 & 8 & $9(28.1 \%)$ \\
\hline Menstrual changes & Nil & 11 & $11(34.37 \%)$ \\
\hline Voice change & 1 & 3 & $4(12.5 \%)$ \\
\hline Depression & 1 & 5 & $6(18.75 \%)$ \\
\hline Neck swelling & Nil & 3 & $3(9.3 \%)$ \\
\hline
\end{tabular}

On physical examination oedema feet $(40.62 \%)$ and pallor $(37.5 \%)$ was the most common finding while delayed deep tendon reflex was present in $15.62 \%$. According to JNC VII guidelines most of the patients had presented us in pre-hypertension stage $(56.25 \%)$ while $31.25 \%$ were non hypertensive. 9.3\% had systolic blood pressure more than $140 \mathrm{~mm}$ of mercury and $12.5 \%$ had diastolic blood pressure more than $90 \mathrm{~mm}$ of mercury. Table 2 shows the thyroid and lipid profile of hypothyroid patients and controls.

Table 2: Comparison of thyroid and lipid profile between two groups

\begin{tabular}{|l|c|c|c|}
\hline Thyroid profile & Hypothyroid & Controls & P-value \\
\hline TSH mlU/ml & $30.98 \pm 33.06$ & $3.25 \pm 0.76$ & \multirow{2}{*}{$<0.0001, \mathrm{HS}$} \\
\cline { 1 - 3 } T3ng/dl & $96.78 \pm 42.06$ & $126 \pm 19.50$ & \\
\hline T4 $\mu \mathrm{g} / \mathrm{dl}$ & $4.99 \pm 1.85$ & $7.36 \pm 0.87$ & \\
\hline Lipid profile $(\mathrm{mg} / \mathrm{dl})$ & \multicolumn{3}{|c|}{} \\
\cline { 1 - 3 } Serum cholesterol & $245.34 \pm 47.31$ & $142.86 \pm 8.65$ & \multirow{2}{*}{$<0.0001, \mathrm{HS}$} \\
\cline { 1 - 3 } Serum triglyceride & $229.40 \pm 50.58$ & $155.53 \pm 6.64$ & \\
\hline Serum LDL & $117.96 \pm 21.75$ & $82.33 \pm 8.65$ & \\
\hline
\end{tabular}

Among the patients presented to us, sinus bradycardia was the commonest electrocardiogram abnormality being present in $43.75 \%$, followed by low voltage complex, ST-T changes and right bundle branch block while $18.75 \%$ patients had normal electrocardiogram (Table 3).

Table 3: Electrocardiographic changes in hypothyroid patients

\begin{tabular}{|l|c|c|c|}
\hline ECG changes & Male & Female & Total \\
\hline Sinus bradycardia & 1 & 13 & $14(43.75 \%)$ \\
\hline Low voltage complex & 1 & 5 & $6(18.75 \%)$ \\
\hline ST-T changes & Nil & 5 & $5(15.62 \%)$ \\
\hline RBBB* & Nil & 1 & $1(3.1 \%)$ \\
\hline Ectopics/Arrythmias & Nil & Nil & Nil \\
\hline Normal & Nil & 6 & $6(18.75 \%)$ \\
\hline
\end{tabular}

There was no statistical difference $(p>0.05)$ in basic two dimensional echocardiographic parameters except for interventricular septal and posterior wall thickness that show significantly raised interventricular septal $(9.6 \pm 1.22)$ and posterior wall thickness $(9.71 \pm 1.08)$ as compared to controls. Also there was no statistically significant difference between mean left ventricular ejection fraction (LVEF) in patients of hypothyroid and controls.

When Doppler parameters were compared, hypothyroid patients showed significant reduction in early diastolic filling $\mathrm{E}(71.19 \pm 6.09 \mathrm{~cm} / \mathrm{sec})$ as compared to controls $(81.53 \pm 9.65 \mathrm{~cm} / \mathrm{sec})$. Conversely, hypothyroid patients shows significantly raised late diastolic filling A $(64.32 \pm 7.74)$ as compared to controls $(50.53 \pm 4.03)$ resulting in 
reduced E/A ratio in hypothyroid $(1.11 \pm 0.1)$ as compared to controls (1.62 \pm 0.248$)$. Similarly, when Tissue Doppler parameters were compared, hypothyroid patients showed significant reduction in peak early diastolic velocity (E') $(7.67 \pm 1.32)$ while peak late velocity (A') was increased in hypothyroid patients $(11.23 \pm 0.91)$ and also shows raised in E/E' (9.51 \pm 1.59$)$ as compared controls. Difference between systolic velocities was not significant (Table 4).

Table 4. Doppler parameter

\begin{tabular}{|l|c|c|c|}
\hline Parameter & Hypothyroid & Controls & p value \\
\hline E $(\mathrm{cm} / \mathrm{sec})$ & $71.19 \pm 6.09$ & $81.53 \pm 9.65$ & $0.0001, \mathrm{HS}$ \\
\hline $\mathrm{A}(\mathrm{cm} / \mathrm{sec})$ & $64.32 \pm 7.74$ & $50.53 \pm 4.03$ & $<0.0001, \mathrm{HS}$ \\
\hline E/A & $1.11 \pm 0.1$ & $1.62 \pm 0.24$ & $<0.0001, \mathrm{HS}$ \\
\hline $\mathrm{E}^{\prime}(\mathrm{cm} / \mathrm{sec})$ & $7.67 \pm 1.32$ & $11.72 \pm 0.97$ & $<0.0001, \mathrm{HS}$ \\
\hline $\mathrm{A}^{\prime}(\mathrm{cm} / \mathrm{sec})$ & $11.42 \pm 0.82$ & $10.76 \pm 1.07$ & $0.0243, \mathrm{~S}$ \\
\hline E/E' & $9.51 \pm 1.59$ & $7.02 \pm 1.07$ & $<0.0001, \mathrm{HS}$ \\
\hline DT $(\mathrm{msec})$ & $186.9 \pm 34.87$ & $179.8 \pm 12.35$ & 0.4494 \\
\hline IVRT $(\mathrm{msec})$ & $101.21 \pm 13.28$ & $81.26 \pm 4.99$ & $<0.0001, \mathrm{HS}$ \\
\hline $\begin{array}{l}\text { SYST } \\
(\mathrm{cm} / \mathrm{sec})\end{array}$ & $9.06 \pm 1.25$ & $9.01 \pm 0.73$ & $0.9337, \mathrm{NS}$ \\
\hline EF\% & $61 \pm 7.1$ & $61 \pm 2.06$ & $1.0, \mathrm{NS}$ \\
\hline
\end{tabular}

Figure 1: Comparison of E/E' ratio between cases and controls

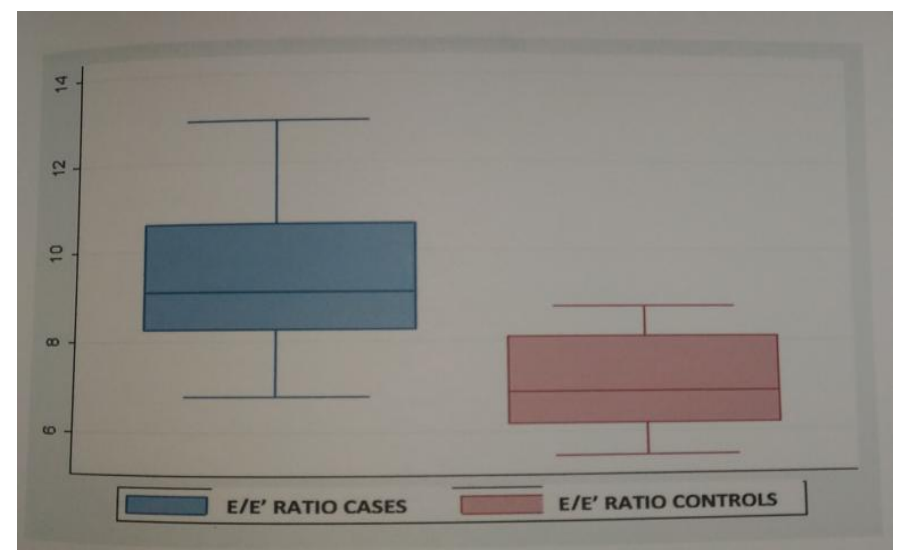

Figure 2: Comparison of IVRT between cases and controls

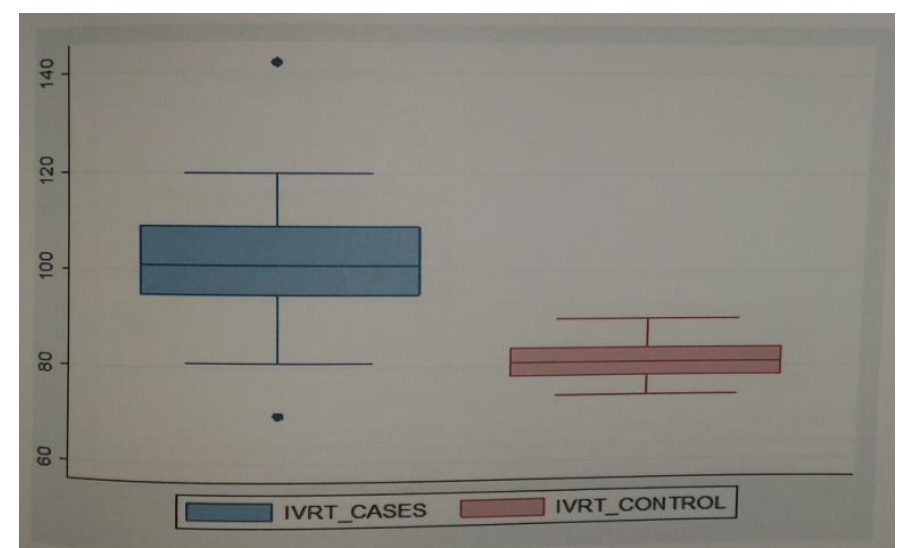

Aforementioned findings suggest significant diastolic dysfunction. No evidence of pulmonary hypertension was present in study group and pericardial effusion was present in two patients of overt hypothyroidism.

\section{Discussion}

The present study was carried out on thirty two hypothyroid patients with aim to assess echocardiography changes and compare these changes with fifteen healthy age and sex matched controls. The mean age of hypothyroid patients was $40.90 \pm 8.73$ years while the mean age of controls was $40.06 \pm 9.68$ years. The literature review ${ }^{[5-7]}$ found various differences in mean age of patients; this might be due to racial variation. Among 32 hypothyroid patients $6.25 \%$ were males and $93.75 \%$ were females while among 15 controls $13.33 \%$ were males and $86.66 \%$ were females, suggesting that female preponderance in our study. Most of the other studies ${ }^{[6-8]}$ conducted had also female preponderance suggesting disease being more common in female population. Out of 32 hypothyroid patients 18 (56.2\%) were subclinical while $14(43.75 \%)$ patients presented with us as an overt hypothyroid.

The weight gain was most commonest symptom observed in hypothyroid patients followed by easy fatigability, dry skin, menstrual disturbance, constipation, depression, voice change and least of all neck swelling. Oedema feet and pallor was the most common finding on physical examination. When patients blood pressure were compared, $59.37 \%$ patients had systolic blood pressure in the range $120-139 \mathrm{~mm} \mathrm{Hg}$, followed by $31.25 \%$ patients $<120 \mathrm{~mm} \mathrm{Hg}$ and $9.3 \%$ patients in the range $140-159 \mathrm{~mm} \mathrm{Hg}$. $56.25 \%$ patients had diastolic blood pressure in the range $80-89 \mathrm{~mm}$ $\mathrm{Hg}$, followed by $31.25 \%$ patients $<80 \mathrm{~mm} \mathrm{Hg}$ and $12.5 \%$ patients in the range $90-99 \mathrm{~mm} \mathrm{Hg}$. None of the patient in our study had blood pressure above $160 / 100$. The present study correlated with other studies ${ }^{[9-11]}$.

As expected, hypothyroid patients had significantly raised $\mathrm{TSH}$ and low $\mathrm{T} 3$ and $\mathrm{T} 4$ as 
compared to controls. When subclinical and overt hypothyroid group compared, overt hypothyroid patients had significantly raised TSH $(58.83 \pm 33.47)$ and low T3 $(54.21 \pm 19.35)$ and T4 (3.2 \pm 1.11$)$ levels as compared to subclinical hypothyroid, (TSH- $9.31 \pm 0.54 \mathrm{mlU} / \mathrm{ml}$, T3$129.88 \pm 16.96 \mathrm{ng} / \mathrm{dl}$ and T4- $6.39 \pm 0.64 \mu \mathrm{g} / \mathrm{dl})$. When lipid profile of hypothyroid and controls were compared, we found significantly raised serum cholesterol, serum triglyceride and serum LDL concentration in hypothyroid as compared to controls. The aforementioned data suggest that hypothyroidism may have a critical role on deterioration of the atherogenic profile. This finding of our study was compared with different studies $^{[12-14]}$. The sinus bradycardia was commonest ECG change observed in $43.75 \%$ patients followed by low voltage complexes, ST-T changes and right bundle branch block while $18.75 \%$ had normal ECG, this was compare with previous studies ${ }^{[15,16]}$.

Among the various M-mode parameters that were evaluated and compared with controls, we found that hypothyroid patients did not show significant difference in left ventricular dimensions other than interventricular septal and posterior wall thickness, that showed significantly raised interventricular septal $(9.6 \pm 1.22)$ and posterior wall thickness $(9.71 \pm 1.08)$ as compared to controls interventricular septal $(7.6 \pm 0.63)$ and posterior wall thickness $(7.56 \pm 0.67)$. Various studies ${ }^{[8,17,18]}$ have shown alteration in myocardial wall thickness in patients of hypothyroidism. The literature shows significantly high incidence of asymmetrical septal or concentric hypertrophy in these patients.

When Doppler parameters were compared hypothyroid patients show significant reduction in early diastolic filling "E wave" as compared to controls, this finding was suggestive of reduced early diastolic filling in hypothyroid patients. In contrast, study group shows significantly raised late diastolic filling "A wave" this resulting in reduced E/A ratio in hypothyroid as compared to controls suggesting augmented atrial contribution to diastolic filling and reduced early diastolic filling or in other words impaired left ventricular diastolic function. It must be realized that reduction in early filling in absence of augmented atrial filling indicates only early and mild diseases, but in our case reduction in early filling was accompanied by augmentation of atrial filling indicating significant diastolic dysfunction. These were significant prolongation of isovolumic relaxation time in hypothyroid patients $(101.21 \pm 13.28)$ as compared to controls $(81.26 \pm 4.99)$ while though the deceleration time was being prolonged it was not significant. The ejection fraction does not significantly vary between two groups. Our findings were correlated with various studies ${ }^{[6,7,13]}$.

In our study, there was significant reduction in peak early diastolic velocity (E') and increased in peak late diastolic velocity (A') were observed in hypothyroid patients as compared to controls. Hypothyroid patients also showed significantly raised E/E' as compared to controls but difference between systolic velocities was not significant; these findings were compared with previous studies $^{[19-21]}$. Discrepancies, among different studies, about diastolic function abnormalities in hypothyroidism can be attributed to difference in selection of age, sex, distribution and inclusion of patients with previous hyperthyroidism and to different diagnostic criteria.

When subclinical and overt hypothyroid patients were compared there was significant increased in interventricular septal diameter in overt $(10.12 \pm 1.29 \mathrm{~mm})$ as compared to subclinical $(9.33 \pm 1.02 \mathrm{~mm})$ though the diastolic dysfunction was present in both groups, the difference was not statistically significant. The pericardial effusion was observed only in overt hypothyroid patient. Our results were correlated with other studies $[8,13,18]$. When we compared different diastolic parameters we did not found any statistically significant difference in relation to age and hypertension. So the diastolic dysfunction seen in study group can be attributed to hypothyroid state per se, by removing the confounding factor 
hypertension and age, which itself can act as a risk factor for diastolic dysfunction through matching.

\section{Limitations}

There were some limitations of the study which includes -1 . Patients could not be fallowed up to look for the resolution of cardiac changes after achievement of euthyroid state, 2. Free T3 and T4 levels could not be done due to financial constraints, 3. Other parameters like myocardial performance index, left atrial volume estimation would have added to better evaluation of cardiac functions.

\section{Conclusion}

The present study demonstrated that the cardiovascular risk increases in patients with hypothyroidism and several potential risk factors were also similarly reported in patients with subclinical hypothyroidism. Untreated overt hypothyroidism can result in profound cardiac consequences, notably pericardial effusions and congestive heart failure. Fortunately, these clinical sequelae are rare in the modern era of thyroid function testing and early intervention. While an impaired left ventricle diastolic dysfunction characteristically slows myocardial relaxation and impaired ventricular filling is the most consistent abnormality in subclinical hypothyroidism.

The result of this study shows that diastolic function is impaired in patients with hypothyroidism. Doppler- echocardiography may be considered a reliable method for a cross sectional and longitudinal assessment of left ventricular diastolic function in patient with hypothyroidism.

\section{Acknowledgement}

The authors would like to thank the Department of Medicine and administration of Government Medical College, Nagpur, Maharashtra, for permission to study and providing facility to carry out the work.

\section{References}

1. Stiefelhagen P. Cardiac symptoms in endocrinologic disorders. Heart illness caused by the thyroid gland. MMW. Fortschr. Med. 2009; 151(19):12-13.

2. Brutsaert DL, Sys SU, Gilleberi TC. Diastolic failure: Pathophysiology and therapeutic implications. J Am Coll Cardiol 1993;22:318-125.

3. Klein I, Ojama K. Thyroid hormone and cardiovascular system. N Engl J Med 2001;344:501-509.

4. Rodondi N, Bauer DC, Cappola AR. Subclinical thyroid dysfunction, cardiac fuction and the risk of heart faire. The cardiovascular Health study. J Am. Coll. Cardiol 2008;52(14):1152-1159.

5. Biondi B, Fazio S, Palmieri EA. Left ventricular diastolic dysfunction in patients with subclinical hypothyroidism. J. Clin Endocrinol Metab 1999;84:20642067.

6. Mishra TK, Routray SN, Das S, Behera M. Left ventricular dysfunction in patients with subclinical hypothyroidism and its reversibility after hormone therapy 2005;53:943-946.

7. Meena CL, Meena RD, Nawal R, Meena VK, Bharti A , Meena LP. Assessment of left ventricular diastolic dysfunction in subclinical hypothyroidism. Acta Inform Med 2012;20(4):218-220.

8. Rawat B, Satyal A. An echocardiographic study of cardiac changes in hypothyroidism and the response to treatment. Kathmandu Univ Med J 2003;2(3):182-187.

9. Saito I, lto K, Saruta T, Hypothyroidism as a cause of hypertension. Hypertension 1983;5(1):112-115.

10. Fommei E, Lervasi G. The role of thyroid hormone in blood pressure homeostasis: evidence from short term hypothyroidism in human . J. Chin. Endocrinol. Metab 2002;87:1996-2000. 
11. Kotsis V, Alevizaki M, Stabouli S. Hypertension and hypothyroidism: results from an ambulatory blood pressure monitoring study. J Hypertens 2007;25:993-999.

12. William J, Hueston MD, William S, Pearson MHA. Subclinical hypothyroidism and the risk of hypercholesterolemia. Ann Fam Med 2004;2(4):351-355.

13. Gupta A, Sinha RS. Echocardiographic changes and alterations in lipid profile in cases of subclinical and overt hypothyroidism. J Assoc Physiciar S India 1996;44(8):546,551-553.

14. Canaries GJ. The Colorado thyroid disease prevalence study. Arch Intern Med 2000;160(4):526-534.

15. Al-Farttoosi. Cardiovascular manifestations of primary hypothyroidism. The Iraqi postgraduate medical jpurnal 2010;9(2):113-119.

16. Agarwal. Electrocardiographic changes in patients with hypothyroidism. Endocrine Abstracts 2004;7:246.

17. Varma R, Jain AK, Ghose T. Heart in hypothyroidism-an echocardiographic study. J Assoc Physicians India 1996;44(6):390-392.

18. Galderisi M, Vitale G, D'Errico A et al. Usefulness of pulsed tissue Doppler for the assessment of left ventricular myocardial function in overt hypothyroidism, Ital Heart J 2004;5(4):257-264.

19. Gulbanu Erkan, Aycan Fahri Erkan, Atiya Cengel. The evaluation of diastolic dysfunction with tissue Doppler echocardiography in women with subclinical hypothyroidism and the effect of L-Thyroxine treatment on diastolic dysfunction: A pilot study. J Thyroid Res 2011.2011;654304.
20. Fatma Alibaz Oner, Selen Yurdakul, Ender Oner et al. Evaluation of ventricular function using tissue Doppler echocardiography in patients with subclinical hypothyroidism. Turk Kardiyol Dern Ars-Arch Turk Soc Cardiol 2011;39:129-136.

21. Xin Chen, Na Zhang, Yunfei Cai, Jingpu Shi. Evaluation of left ventricular diastolic function using tissue Doppler echocardiography and conventional Doppler echocardiography in patients with subclinical hypothyroidism age $<60$ years: A meta-analysis. Journal of Cardiology 2013;61(1):8-15. 